Images dans le monde ibérique et ibéricoaméricain

$8 \mid 2015$

L'Histoire et ses récits entre images, fictions et paratextes

\title{
Les Sánchez Muñoz et le Romanz del Cid
}

Étude des marginalia du manuscrit Esp 220 de la Bibliothèque Nationale de France (XV s.)

\section{Patricia Rochwert-Zuili}

\section{(2) OpenEdition}

Journals

\section{Édition électronique}

URL : http://journals.openedition.org/agedor/403

DOI : 10.4000 /agedor.403

ISSN : 2104-3353

Éditeur

Laboratoire LISAA

Référence électronique

Patricia Rochwert-Zuili, « Les Sánchez Muñoz et le Romanz del Cid », L'Âge d'or [En ligne], 8 | 2015, mis en ligne le 01 février 2016, consulté le 03 mai 2019. URL : http://journals.openedition.org/agedor/403 ; DOI : 10.4000/agedor.403

L'Âge d'or. Images dans le monde ibérique et ibéricoaméricain 


\title{
Les Sánchez Muñoz et le Romanz del Cid. Étude des marginalia du manuscrit Esp 220 de la Bibliothèque Nationale de France $\left(\mathrm{XV}^{\mathrm{e}} \mathrm{s}\right.$.)
}

\begin{abstract}
Résumé : À travers l'étude de ses marginalia, on retrace ici l'histoire de l'un des témoignages manuscrits de la Chronique de Castille, de sa composition à sa réception. Les interactions entre le texte, les images et les annotations marginales révèlent non seulement l'identité de celui qui pourrait être le promoteur de cette copie navarro-aragonaise, mais aussi celle de ses divers lecteurs. Cette recherche mène ainsi à la redécouverte de l'unique manuscrit sur l'histoire du Cid que possédaient les Sánchez Muñoz, l'une des familles les plus puissantes de Teruel.
\end{abstract}

Mots-clés : Chronique de Castille - le Cid - Clément VIII - Grand Schisme d'Occident - château de Peñiscola - Sánchez Muñoz - Teruel

Resumen: Mediante el análisis de sus marginalia, se describe aquí la historia de uno de los testimonios manuscritos de la Crónica de Castilla, desde su composición hasta su recepción. Las interacciones entre el texto, las imágenes y las anotaciones marginales no solo revelan la identidad de quien podría ser el promotor de la copia, sino también la de sus distintos lectores. Este trabajo de investigación desemboca pues en el redescubrimiento del único manuscrito sobre la historia del Cid que poseían los Sánchez Muñoz, una de las familias más potentes de Teruel.

Palabras clave: Crónica de Castilla - el Cid - Clemente VIII - Gran Cisma de Occidente - castillo de Peñíscola - Sánchez Muñoz - Teruel

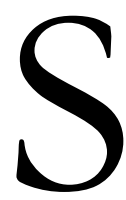

i l'étude de la riche matière narrative des chroniques médiévales offre de précieuses informations sur les propos de ceux qui furent à l'origine de la composition de ces textes, l'observation des manuscrits et de leurs marginalia réserve parfois de jolies découvertes sur le destin de ces œuvres. Composée dans les premières années du $\mathrm{XIV}^{\mathrm{e}}$ siècle, la Chronique de Castille nous est conservée dans dix-neuf manuscrits datant, pour la plupart, du $\mathrm{XV}^{\mathrm{e}}$ siècle ${ }^{1}$, qui témoignent du succès incontestable dont l'œuvre fit l'objet. Le caractère imposant de cette tradition manuscrite s'explique sans doute par l'éclairage exclusif que porte la chronique sur le règne des premiers rois de Castille, de Ferdinand $\mathrm{I}^{\mathrm{er}}$ (1035-1065) à Ferdinand III (1217/1230-1252), et par la place de choix qu'elle accorde au récit des hauts faits du Cid fondé, notamment, sur deux poèmes épiques : la Chanson de Rodrigue et la Chanson de mon

\footnotetext{
${ }^{1}$ Pour une description de la vingtaine de manuscrits qui composent la tradition manuscrite de la Chronique de Castille, voir en particulier ALVAR, Carlos et LUCÍA MEGÍAS, José Manuel (coord.), Diccionario filológico de literatura medieval española. Textos y transmisión, Madrid, Castalia, 2002, p. 286-290. On trouvera une étude et une édition de la chronique, réalisée à partir de deux manuscrits, dans ROCHWERT-ZUILI, Patricia, Crónica de Castilla, Les Livres d'e-Spania, «Sources », 1, Paris, SEMH-Sorbonne - CLEA (EA 4083), 2010.
} 
Cid. Or, parmi ces manuscrits, il en est un qui se distingue par sa singularité : le manuscrit Esp. 220 de la Bibliothèque Nationale de France. Membre de la deuxième famille de manuscrits, marquée par l'accentuation du caractère romanesque du récit et une plus grande liberté dans la formulation des titres de chapitres, il est non seulement écrit en navarro-aragonais et illustré par une trentaine de dessins, dont certains débordent largement sur les marges, mais il présente surtout un nombre considérable d'annotations marginales de nature et de longueur variables.

Composé de 168 folios, le manuscrit Esp 220 mesure 275 x 205 mm. Sa couverture, de couleur blanche, est décorée par un losange. Le texte est écrit sur deux colonnes par deux mains distinctes dont l'écriture est une gothique rotunda ${ }^{2}$. Après un sommaire occupant les premiers folios, on découvre, au folio $12 \mathrm{r}^{\circ}$, les premières lignes du récit qui s'ouvre sur un titre en rouge indiquant «Aqui comiença el romanz del Cid Campeador e de los reyes de Castiella ${ }^{3}$ et qui s'achève au fol. $167 \mathrm{v}^{\circ}$ a sur ces mots : "Acabado es este romanz del noble Cid Campeador e de las conquistas de Castiella, el qual libro scriuio [illisible] escrito fue en el anno mill $\operatorname{cccc}^{\text {os }}$ xxviiii, el qual libro es de [illisible]».

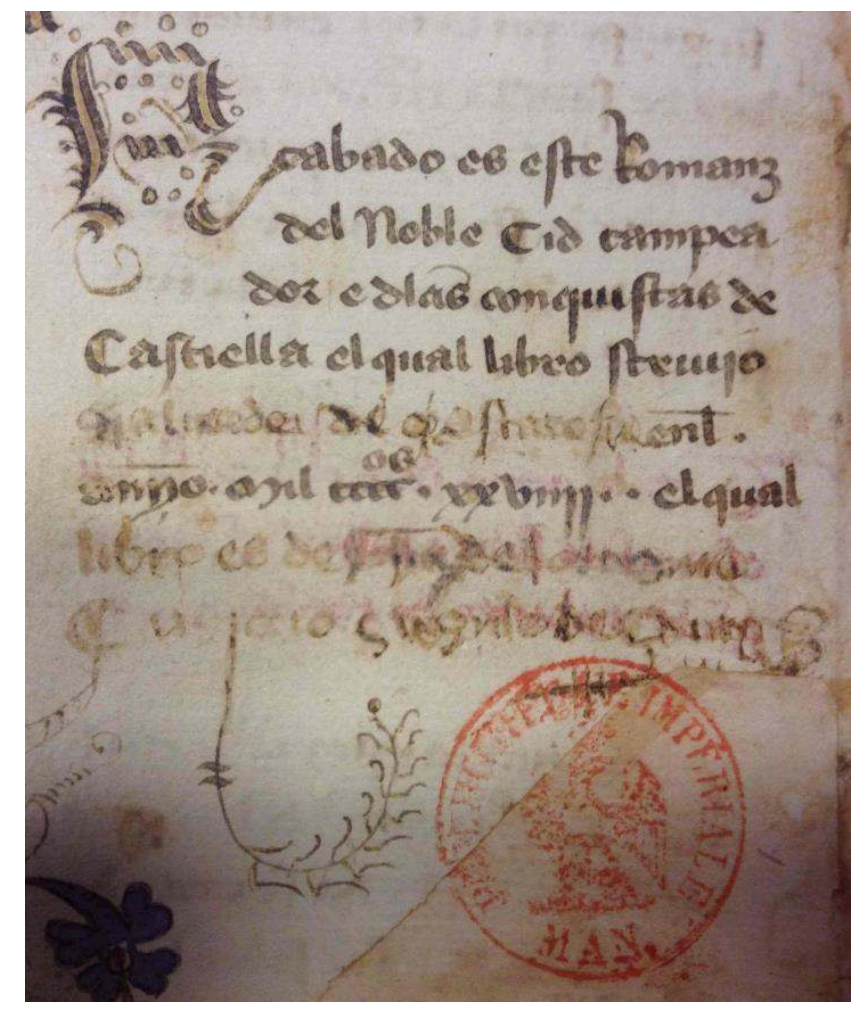

Fol. $167 \mathrm{v}^{\circ}(\mathrm{ms}$. Esp 220, BnF)

Même si l'identité du copiste et celle du premier propriétaire du manuscrit ont été effacées, ces quelques lignes nous renseignent sur les éléments du récit qui ont justifié la confection du manuscrit et sur la date de son achèvement, 1429, ce qui concorde avec son écriture. Cependant, on trouve en marge du texte, au folio $167 \mathrm{v}^{\circ} \mathrm{b}$, une indication réalisée avec une encre plus claire et une écriture quelque peu différente qui tendrait à brouiller les pistes : « este libro es de Gaspar Johan Sanches Munyoz, escriuiose en anyo mill cccc xxxxvi a xx ocho de abril vispera san Pedro martir ». En effet, si cette information marginale rattache ce manuscrit à l'Aragon, Gaspar Johan

\footnotetext{
${ }^{2}$ L'écriture que l'on trouve aux folios $20 \mathrm{v}^{\circ}-22 \mathrm{v}^{\circ}$ et $25 \mathrm{r}^{\circ} \mathrm{a}-27 \mathrm{v}^{\circ}$ est en effet différente de celle qui domine dans le manuscrit.

${ }^{3}$ Les citations ont été transcrites en résolvant les abréviations. La ponctuation et l'usage des majuscules obéissent aux règles actuelles. Il en est de même pour l'union et la séparation des formes. L'apostrophe indique les cas d'élision ou de contraction. Les chevrons $<>$ sont utilisés pour combler les passages lacunaires.
} 
Sánchez Muñoz étant issu de l'une des familles les plus puissantes de Teruel ${ }^{4}$, elle mentionne non seulement une autre date de composition, mais nous renvoie surtout au tournant des $\mathrm{XV}^{\mathrm{e}}$ et $\mathrm{XVI}^{\mathrm{e}}$ siècles, puisque cet homme vécut de 1483 à 1543. Cela suscite donc toute une série de questions. Gaspar Johan Sánchez Muñoz pourrait-il être l'auteur (ou l'un des auteurs) des annotations marginales que l'on trouve dans le manuscrit? Qu'est-ce qui, dans la nature et le propos de ces annotations, permettrait de le supposer? Dans quelle mesure les annotations marginales pourraient-elles révéler l'histoire de ce manuscrit, de sa composition à sa réception?

Pour répondre à ces interrogations, j'évoquerai d'abord quelques aspects du contexte de composition du manuscrit Esp 220 et je m'intéresserai aux choix opérés par l'auteur des illustrations. Je tenterai ensuite d'ébaucher une typologie des annotations et de décrire les interactions entre le texte, les images et les annotations marginales, ce qui me mènera, enfin, à formuler des hypothèses sur le lieu où le manuscrit aurait été composé et sur l'identité de son promoteur et de ses lecteurs.

\section{Le texte et ses illustrations : description et contextualisation}

Afin de comprendre pourquoi, dans les années $20 \mathrm{du} \mathrm{XV}^{\mathrm{e}}$ siècle, on réalisa une copie navarro-aragonaise d'une chronique destinée à conforter le pouvoir de la royauté castillane et à célébrer les hauts faits du $\mathrm{Cid}^{5}$, il convient d'abord de remonter quelques années en arrière. En effet, en 1412 le Compromis de Caspe avait porté sur le trône d'Aragon, en la personne de Ferdinand d'Antequera, la dynastie Trastamare. Or, en accédant au pouvoir, Ferdinand avait jeté les bases d'une union des royaumes péninsulaires à travers une savante politique matrimoniale susceptible, notamment, d'assurer une place et un rôle politique d'importance à ses enfants en Castille : son fils aîné, Alphonse, le futur Alphonse V le Magnanime (1416-1458) avait été marié à Marie de Castille, Jean, son second fils, qui allait régner sur la Navarre à partir de 1425 grâce à son mariage avec Blanche, lui avait succédé à la tête du duché de Peñafiel, et Henri et Sanche étaient devenus respectivement Maîtres des Ordres de Saint-Jacques et d'Alcantara. Mais en 1429, date de la réalisation du manuscrit, Jean de Navarre et son frère Henri avaient dû renoncer à leurs prétentions castillanes face au clan d'Alvaro de Luna et de Jean II, soutenu par une société castillane qui considérait les infants d'Aragon comme des étrangers. Aussi, en mars 1429, Alphonse V le Magnanime avait-il envahi la Castille avec l'aide de son frère Jean, une entreprise qui s'était rapidement soldée par un échec ${ }^{6}$.

Tout porterait donc à croire que cette copie de la Chronique de Castille était destinée à affirmer le pouvoir d'Alphonse $\mathrm{V}$ en Aragon, mais aussi à légitimer son action et à revendiquer les droits que détenaient les infants d'Aragon en Castille, voire, peut-être, à conforter la place de son frère Jean sur le trône de Navarre. Sur ce point, la figure cidienne était d'ailleurs un objet de propagande idéal. Le Cid était non seulement le héros emblématique de la Castille, mais il avait aussi marié ses filles à des infants d'Aragon et de Navarre et était l'ancêtre du Restaurateur de la dynastie navarraise.

\footnotetext{
${ }^{4}$ Sur les Sánchez Muñoz, on pourra consulter NAVARRO ESPINACH, Germán, « Muñoces, Marcillas y otras familias dominantes en la ciudad de Teruel (1435-1500) », Anuario de Estudios Medievales, 32(2), 2002, p. 723-776 et en particulier p. 734-743. Voir aussi MUÑOZ GARRIDO, Vidal, «El linaje de los Sánchez Muñoz en Teruel (11701500) », Aragón en la Edad Media, 17, 2003, p. 263-278.

5 Sur les propos de la Chronique de Castille et sur ceux qui furent à l'origine de sa composition, voir ROCHWERTZUILI, P. (éd.), Crónica de Castilla, Introduction, notamment paragraphes 84-85.

${ }^{6}$ En juillet 1430, Alphonse $V$ fut contraint de signer un traité de paix qui écarta les infants d'Aragon de la Castille, les privant, qui plus est, de tous leurs biens. Sur cette guerre qui opposa les Aragonais au clan de Jean II et d'Alvaro de Luna, voir notamment VALDEÓN BARUQUE, Julio, Los Trastámaras. El triunfo de una dinastía bastarda, Madrid, Temas de hoy, $1^{\text {re }}$ éd., mai 2001, 3e éd., avril 2002, p. 126-144.
} 
Telles sont, du reste, quelques-unes des conclusions auxquelles parvient Ghislaine Fournès dans un article consacré aux illustrations du manuscrit Esp 220, où elle met en lumière l'importance croissante accordée à la figure cidienne dans ces images au point qu'à la série de représentations des monarques castillans se substituent celles du Cid qui, en outre, après sa mort et l'embaumement de son corps, est représenté à plusieurs reprises dans des dessins où figurent aussi les infants d'Aragon et de Navarre lui rendant hommage ${ }^{7}$. Ainsi peut-on suivre la chercheuse lorsqu'elle affirme que le Cid apparait «comme un héros, non plus seulement castillan, mais supranational, assurant la cohésion entre les différents royaumes péninsulaires chrétiens $»^{8}$.

Toutefois, il est un autre aspect de ces miniatures qu'évoque Ghislaine Fournès dans son étude et sur lequel il convient de s'attarder davantage : la représentation de visions et de prodiges. En effet, on remarque que la plupart des épisodes mettant en scène des saints donnent lieu à une illustration. C'est le cas du passage évoquant l'apparition de saint Isidore venu annoncer à Ferdinand $\mathrm{I}^{\mathrm{er}}$ sa mort prochaine, où l'on trouve la première image du manuscrit ${ }^{9}$, mais aussi de celui où saint Pierre, représenté en compagnie d'un ange, annonce au Cid qu'il va mourir ${ }^{10}$. De même trouve-t-on une belle illustration de la bataille qui oppose les hommes du Cid au roi Bucar du Maroc, où l'on distingue, au centre, saint Jacques combattant contre les Maures ${ }^{11}$ :

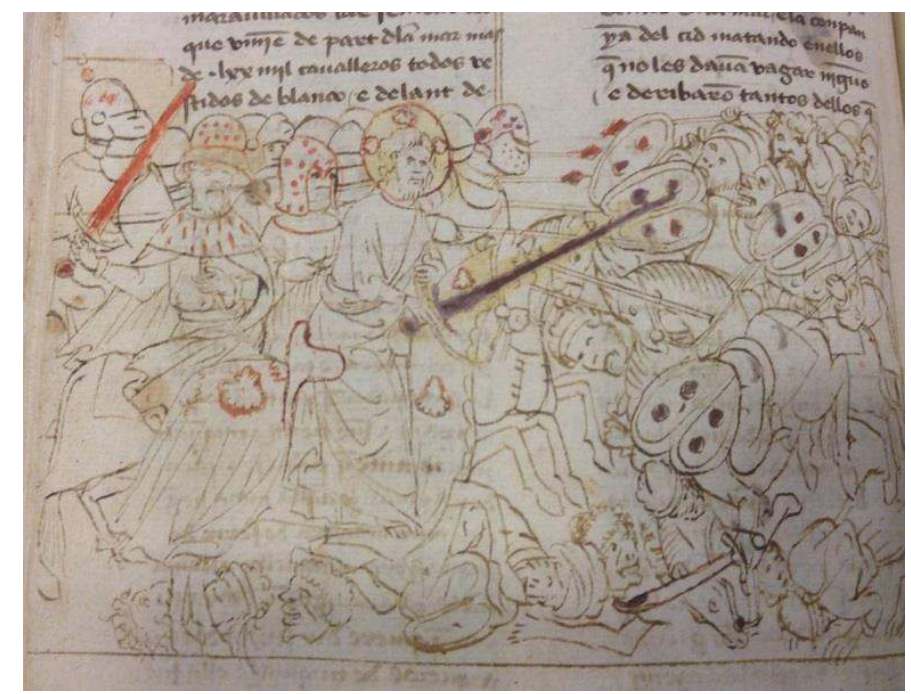

Fol. 126v ${ }^{\circ}$

Or, on note aussi, dans ces images, la présence récurrente des hommes d'église. Ainsi le dessinateur a-t-il pris soin, par exemple, d'illustrer l'épisode où Sanche VI de Navarre, ayant envahi et pillé les terres burgalaises, est stoppé dans son entreprise par les moines de Cardeña qui lui rappellent qu'il est un descendant du Cid, par une image occupant la moitié du folio où l'on voit une troupe de moines à cheval arborant la bannière du $\mathrm{Cid}^{12}$ :

\footnotetext{
${ }^{7}$ FOURNÈS, Ghislaine, «Une histoire castillane en images : les miniatures du manuscrit de Romanz del Cid Campeador $e$ de los reyes de Castiella (1429)", in Image et pouvoir : actes du $4^{e}$ congrès international du GRIMH, 18-19-20 novembre 2004, 4, 2006, p. 111-119.

${ }^{8}$ Ibid., p. 117.

${ }^{9}$ Fol. 28r'.

${ }^{10}$ Fol. 122r ${ }^{\circ}$.

${ }^{11}$ Fol. 126v'.

${ }^{12}$ Fol. $134 r^{\circ}$.
} 


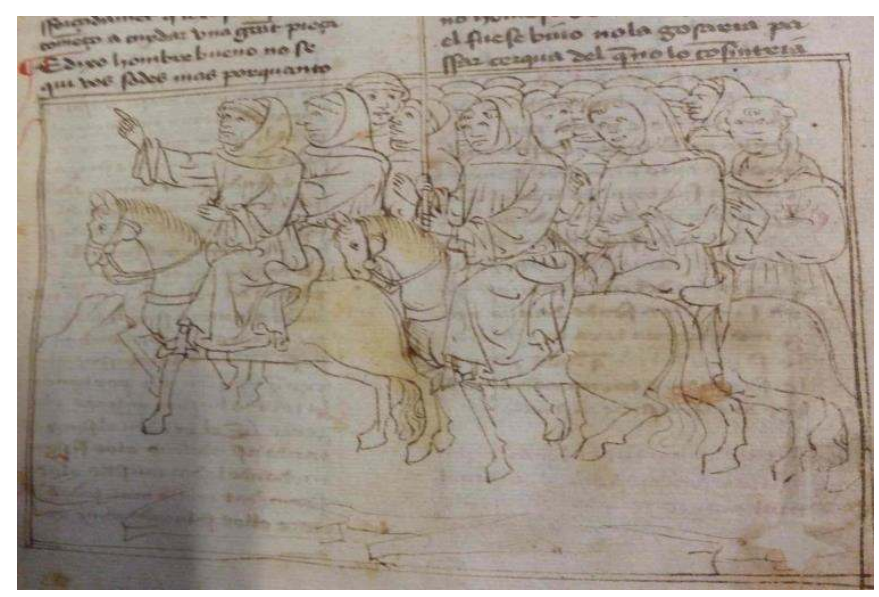

Fol. $134 \mathrm{r}^{\circ}$

On remarque, en outre, que l'évêque Jérome de Valence est représenté à plusieurs reprises dans les miniatures. Il apparaît notamment devant la muraille de Valence face au Cid qui lui confie la garde de la ville avant de se rendre aux cortès de Tolède ${ }^{13}$. De même figure-t-il parmi les hommes se tenant au chevet du Cid mourant ${ }^{14}$. D'ailleurs, dans cette image, l'évêque se distingue des autres personnages par la touche de rouge apposée sur sa mitre :

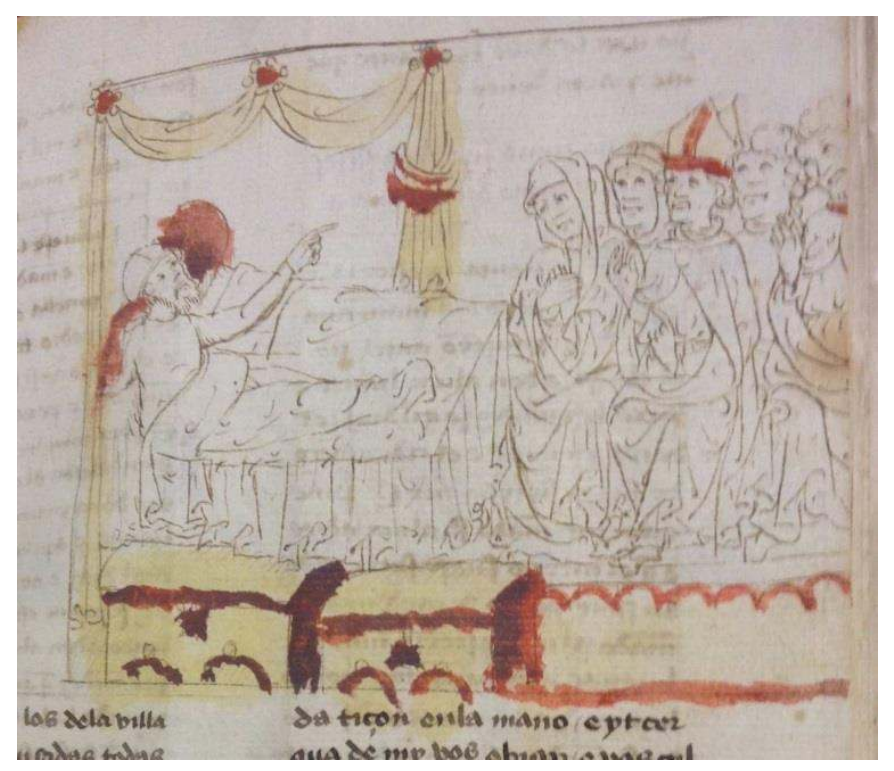

Fol. $124 \mathrm{r}^{\circ}$

On le retrouve, enfin, dans la série d'illustrations représentant le cortège qui mène le corps du Cid placé sur son cheval jusqu'au monastère de Cardeña ${ }^{15}$.

Il convient donc de se demander pourquoi on a accordé une telle importance à ce personnage, d'autant que plusieurs annotations marginales s'attardent aussi sur lui. Dans la première, qui porte sur sa nomination en tant qu'évêque de Valence, apparait d'ailleurs le terme catalan «bisbe »: « este fue el primero bisbe de Valencia porque la conquisto el Cid e fizolo bisbe $»^{16}$ :

\footnotetext{
${ }^{13}$ Fol. $102 \mathrm{r}^{\circ}$.

${ }^{14}$ Fol. $124 \mathrm{r}^{\circ}$.

15 Voir fol. $128 \mathrm{v}^{\circ}$ et $129 \mathrm{v}^{\circ}$.

${ }^{16}$ Fol. $65 \mathrm{r}^{\circ}$.
} 


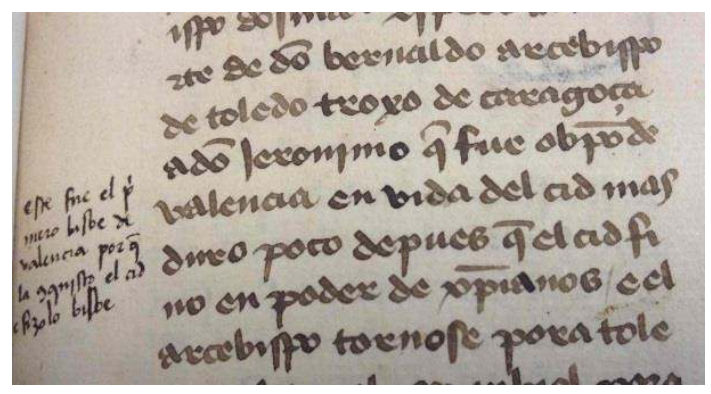

Fol. $65 \mathrm{r}^{\circ}$

La seconde se présente sous la forme d'un trait vertical surmonté de trois points qui signale le passage où est évoquée sa mort $^{17}$ :

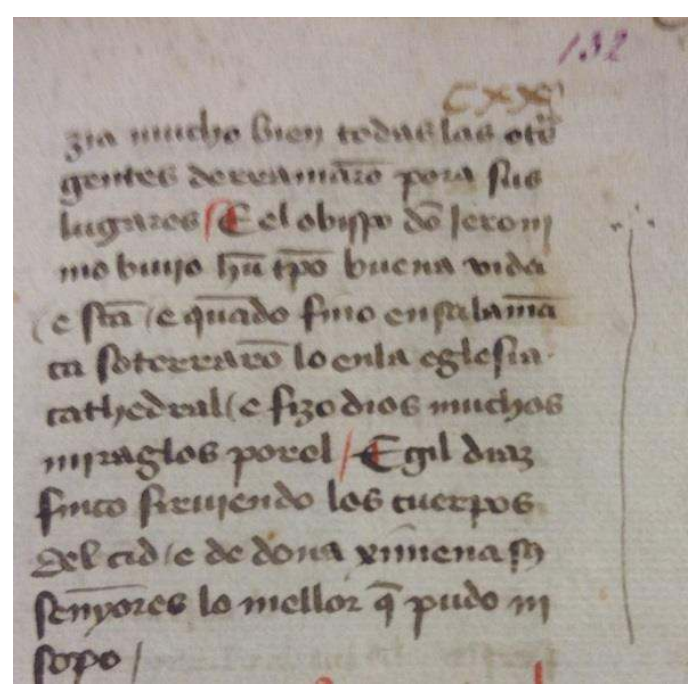

Fol. $132 \mathrm{r}^{\circ}$

Ces éléments sembleraient révéler des coïncidences entre les images et les annotations, ce qui laisserait supposer qu'elles relèveraient d'une même interprétation du texte issue, sans doute, d'un lectorat clérical. Examinons donc à présent les différentes annotations marginales afin d'aller plus loin dans la démonstration.

\section{Typologie et étude des annotations marginales}

Le manuscrit Esp 220 de la Bibliothèque Nationale de France permet d'observer divers types d'annotations, des plus simples, signalant l'importance d'un passage, aux plus élaborées, telles les gloses.

Ainsi trouve-t-on les éléments suivants :

- un o entouré de deux barres obliques $(/ \mathrm{o} /)$ employé généralement devant les titres de chapitres et en particulier devant ceux qui retracent l'histoire du Cid à Valence. Tel est le cas, par

\footnotetext{
${ }^{17}$ Fol. $132 r^{\circ}$
} 
exemple, du passage évoquant le mariage des filles de Rodrigue avec les infants d'Aragon et de Navarre $^{18}$ :

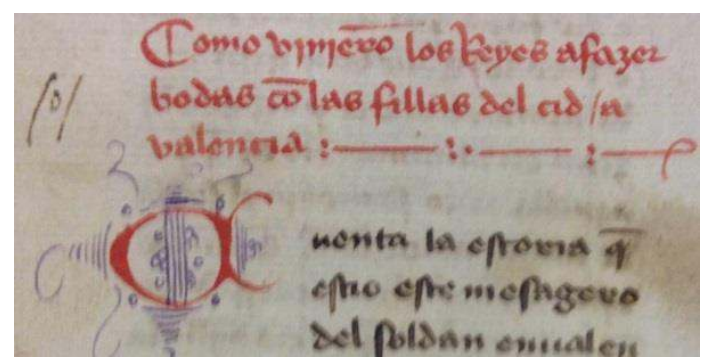

Fol. $118 \mathrm{v}^{\circ}$

Si la plupart de ces chapitres sont centrés sur Valence, certains portent néanmoins sur d'autres faits. C'est ce que l'on peut voir au folio $135 \mathrm{v}^{\circ}$ où est conté le miracle qui s'était produit à Saint-Isidore de León où de l'eau avait jailli des pierres situées devant l'autel de l'église huit jours avant la mort d'Alphonse $\mathrm{VI}^{19}$. De même retrouve-t-on ce signe devant le titre du chapitre annonçant les conquêtes d'Alphonse $\mathrm{VI}^{20}$, mais aussi, détail plus marquant compte tenu de la langue du manuscrit, devant celui qui traite de la guerre qu'Alphonse VII livra à l'Aragon à la mort d'Alphonse $\mathrm{I}^{\mathrm{er}}$ le Batailleur ${ }^{21}$.

- le trait vertical surmonté de trois points - évoqué précédemment - est utilisé aussi pour désigner les passages ayant retenu l'attention du lecteur. Parfois, il s'accompagne d'une précision sur le contenu du récit, comme on peut le voir au folio $143 \mathrm{v}^{\circ}$ où l'on peut lire, en abrégé, «nota miraculum », expression qui signale le moment où saint Isidore apparaît en songe à Alphonse VII pour lui annoncer qu'il l'aidera à prendre Baeza :

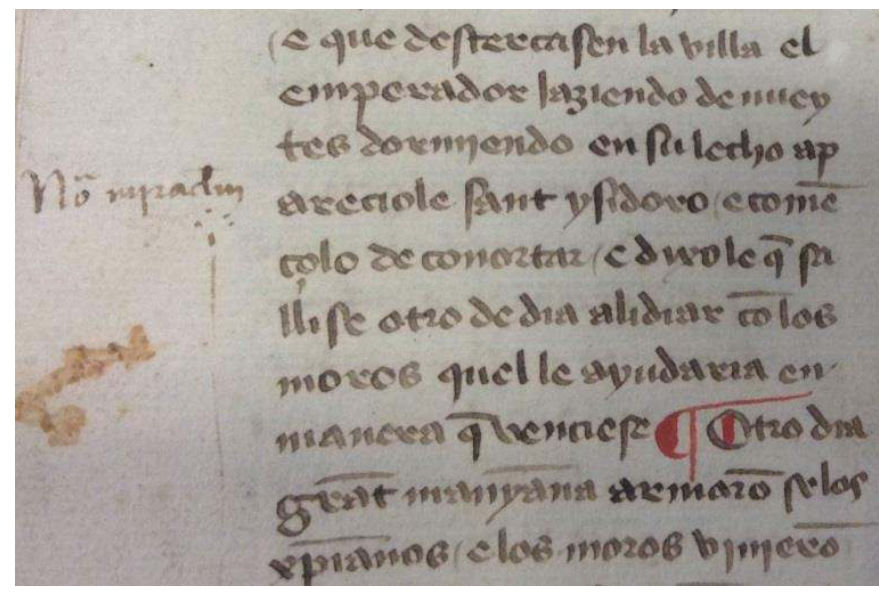

Fol. $143 \mathrm{v}^{\circ}$

- l'exemple précédent permet également d'observer un troisième type d'annotation. En effet, nombreux sont les cas où l'on trouve dans la marge le mot "nota " (voire parfois "nota bene»), abrégé ou pas. Il est souvent placé aux côtés du trait surmonté de trois points pour signaler, comme on l'a vu ci-dessus, des passages relatant des faits miraculeux. On en trouve

\footnotetext{
${ }^{18}$ Fol. $118 v^{\circ}$. Voir aussi fol. $85 v^{\circ}$ et $86 \mathrm{r}^{\circ}$. Dans le dernier exemple, on trouve d'ailleurs dans la marge du folio $86 v^{\circ}$ un trait surmonté de trois points qui met l'accent sur deux des neuf paroisses créées à Valence : San Pedro et Santa María de las Virtudes.

${ }^{19}$ Fol. $135 \mathrm{v}^{\circ}$.

${ }^{20}$ Fol. $70 \mathrm{r}^{\circ}$.

${ }^{21}$ Fol. $141 v^{\circ}$.
} 
d'autres exemples au folio $15 \mathrm{r}^{\circ}$, où apparait l'épisode qui rapporte comment saint Lazare, ayant pris la forme d'un lépreux pour éprouver la charité du Cid, apparait à Rodrigue durant la nuit pour l'assurer d'une destinée prospère ${ }^{22}$, au folio $19 \mathrm{r}^{\circ}$ et $\mathrm{v}^{\circ}$, où le texte évoque l'apparition de saint Jacques à Ferdinand $\mathrm{I}^{\text {er }}$, ou encore au folio $21 \mathrm{r}^{\circ}$, où l'on nous raconte comment Jésus-Christ apparut au pape Léon VII sous la forme d'un pauvre.

- à cela s'ajoutent des commentaires de longueur variable apportant, pour la plupart, des précisions sur le contenu des passages. Certains se composent d'un seul mot, comme c'est le cas d'un passage, copié par la deuxième main du manuscrit, décrivant l'excommunication du pape Benoît IX pour simonie et la nomination de Sylvestre III, où l'on découvre dans la marge le terme « cisma $»^{23}$ qui semble avoir été écrit par le copiste lui-même :

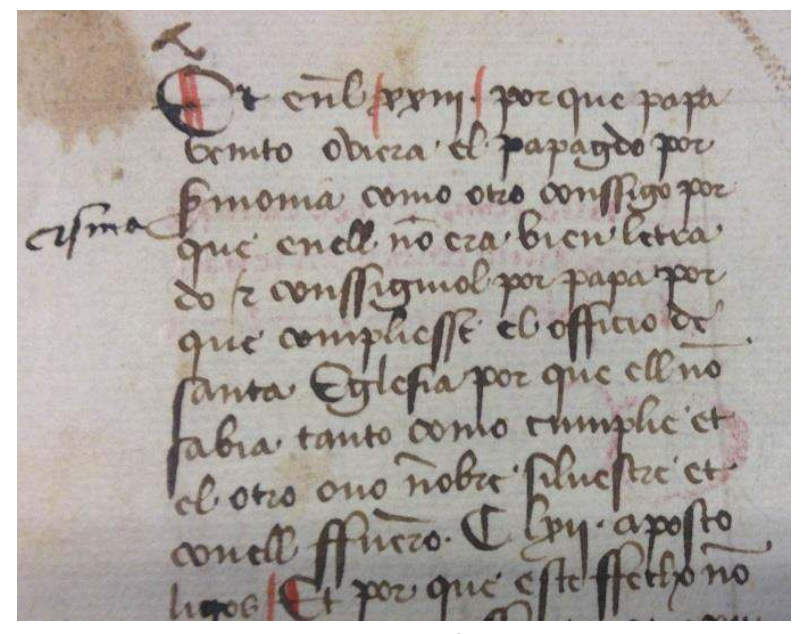

Fol. 20v ${ }^{\circ}$

On trouve aussi des indications toponymiques, comme par exemple l'ajout marginal, au moyen de ce qui s'apparente à un astérisque, de la proposition « que huy es dit lo Puig » associée au château de Cebolla, situé non loin de Valence ${ }^{24}$ :

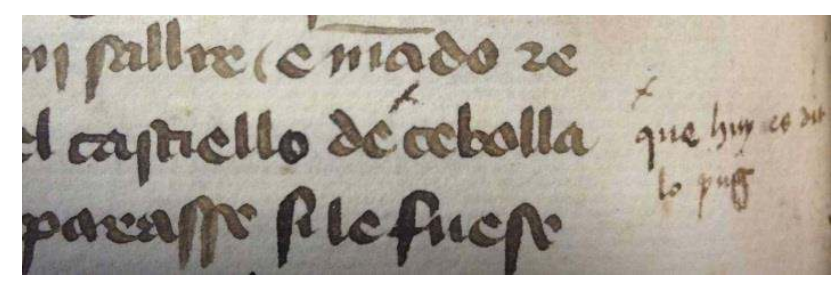

Fol. $71 v^{\circ}$

On relève, par ailleurs, un cas unique de commentaire d'ordre paratextuel. Situé au début du chapitre rapportant le passage du rite mozarabe au rite romain sous le règne d'Alphonse VI, il renvoie au De rebus Hispaniae de l'archevêque de Tolède Rodrigue Jiménez de Rada. En effet, on peut lire au folio $63 \mathrm{v}^{\circ}$ : « que ese capitol [de] la mala reyna [que] queria mudar el oficio mejor lo fallaras en lo libro [de] los godos a xxiiii ${ }^{\circ}$ capitolles ».

\footnotetext{
${ }^{22}$ Dans ce cas, apparaissent d'ailleurs d'autres éléments marginaux, comme l'ajout, au moyen de ce qui s'apparente à un astérisque, de précisions sur les personnages. Ainsi trouve-t-on « el Cid Rui Dias » associé au nom de Rodrigo, ou le terme « lebroso » associé à « el gaffo ».

${ }^{23}$ Fol. 20v ${ }^{\circ}$.

${ }^{24}$ Fol. $71 v^{\circ}$.
} 


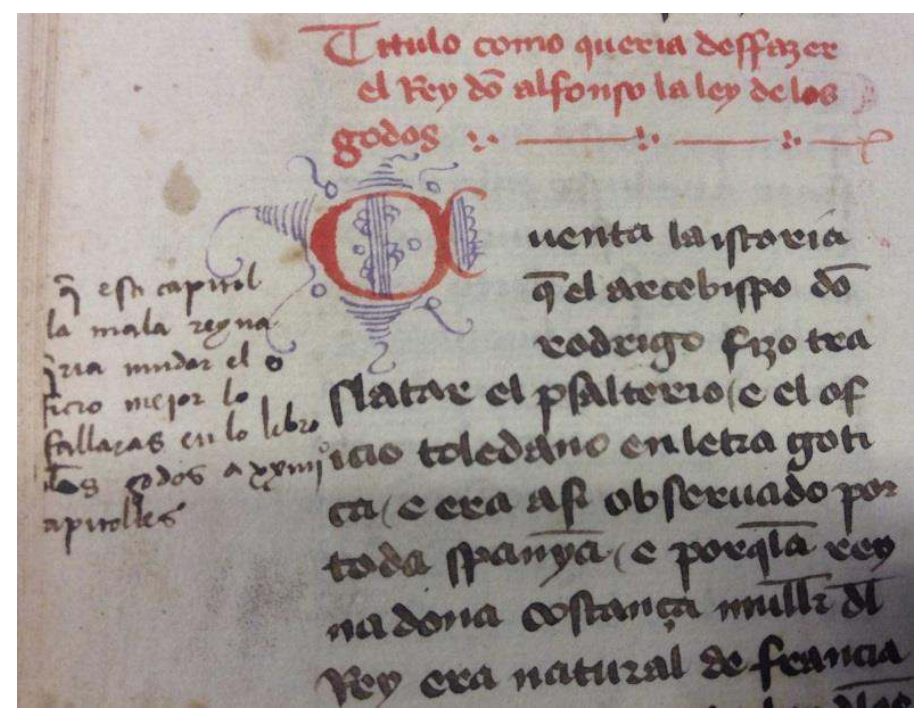

Fol. $63 \mathrm{v}^{\circ}$

Ce commentaire est intéressant à plus d'un titre. D'une part, il porte un jugement personnel sur les faits à travers l'emploi de l'adjectif «mala » et, d'autre part, il montre que le commentateur du texte avait sous la main d'autres livres.

On distingue, enfin, bon nombre de passages où les annotations sont accompagnées de dessins représentant des blasons. Cela se produit systématiquement lorsqu'il est question, dans le texte, d'un personnage portant le nom de Muñoz, de Muñez ou de Núñez parfois corrigés en Muñoz ou Nuñoz. C'est ce que l'on remarque dès le folio $12 \mathrm{v}^{\circ}$ où, au sein du récit de la légende des juges de Castille, est mentionné le nom de la fille de Nuño Rasura, épouse de Laín Calvo, «Eluirea Munyoz ». On trouve en effet dans la marge de gauche le mot nota aux côtés d'un dessin plus foncé représentant un blason divisé en quatre et comportant une croix aux premier et au quatrième quartiers, qui est précisément celui des Muñoz et des Sánchez Muñoz ${ }^{25}$. À cela s'ajoute, dans la marge de droite, un trait vertical surmonté de trois points qui met l'accent sur la descendance d'Elvire et de Laín Calvo, où l'on retrouve, bien entendu, Rodrigue de Vivar.

\footnotetext{
${ }^{25}$ Cf. JULIO DE ATIENZA, Barón de Cobos de Belchite, Nobiliario español. Diccionario heráldico de apellidos españoles y de títulos nobiliarios, Madrid, Aguilar, $2^{\mathrm{e}}$ éd., 1954, p. 560 : «Muñoz. Sus armas : Escudo cuartelado : $1^{\circ}$ y $4^{\circ}$, en campo de oro, una cruz floreteada, de gules, y $2^{\circ}$ y $3^{\circ}$, en campo de oro, tres fajas de gules » et p. $683:$ : Sánchez-Muñoz. Aragonés. De Teruel. Ostentó el Señorío de Escriche y fué uno de los linajes que intervinieron en la conquista de Teruel. [...] Sus armas : Escudo cuartelado : $1^{\circ}$ y $4^{\circ}$, en campo de gules, una cruz floreteada, de oro, y $2^{\circ}$ y $3^{\circ}$, de oro pleno $»$.
} 


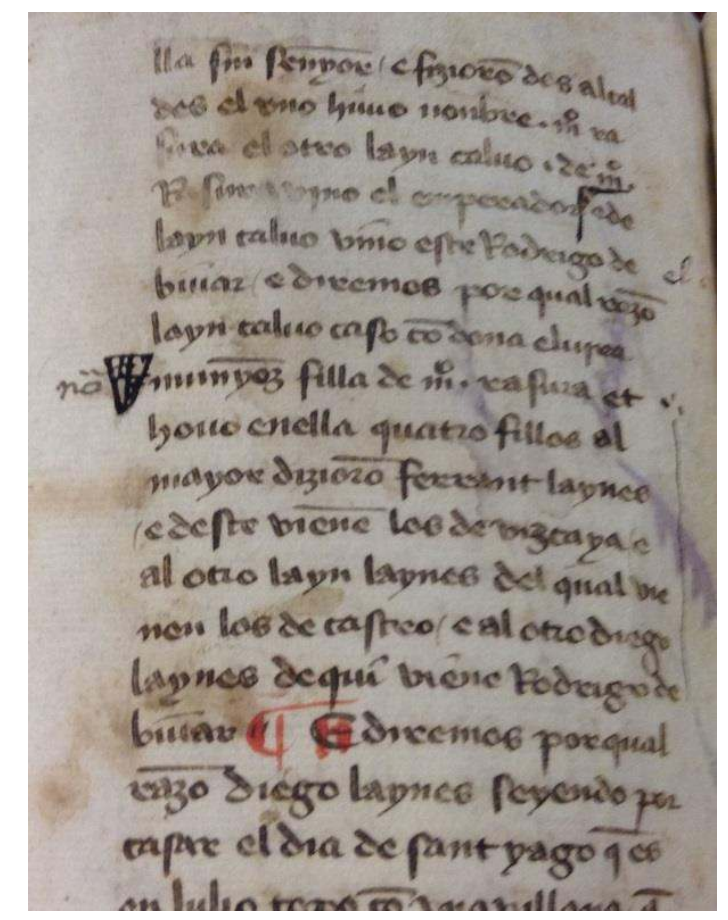

Fol. $12 \mathrm{v}^{\circ}$

$\mathrm{Au}$ folio suivant apparait également un commentaire sur la mère du Cid, "Theresa Nunyoz », où l'on précise, dans une écriture plus tardive qui s'apparente à une gothique précourtisane ou courtisane : «nota de la madre del Cit qual nombre auia »:

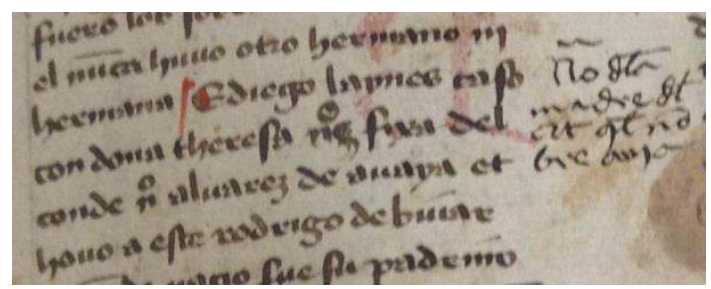

Fol. $13 r^{\circ}$

Cet intérêt pour les Muñoz se manifeste encore au folio $48 \mathrm{v}^{\circ}$ où figure l'énumération des femmes du roi Alphonse VI. Ici, les commentaires ont été réalisés par deux mains distinctes. L'une a introduit, dans une écriture qui s'apparente à celle du manuscrit, une note très courte : " nota de dona Ximena Munyoz». L'autre s'est attardée sur la descendance de cette femme au moyen de deux longs commentaires de nature paraphrastique complétés par un blason qui ressemble au précédent mais dont le bord inférieur est plus arrondi. L'écriture de ces commentaires, plus tardive, est proche de celle que l'on a vue dans la reproduction précédente. 


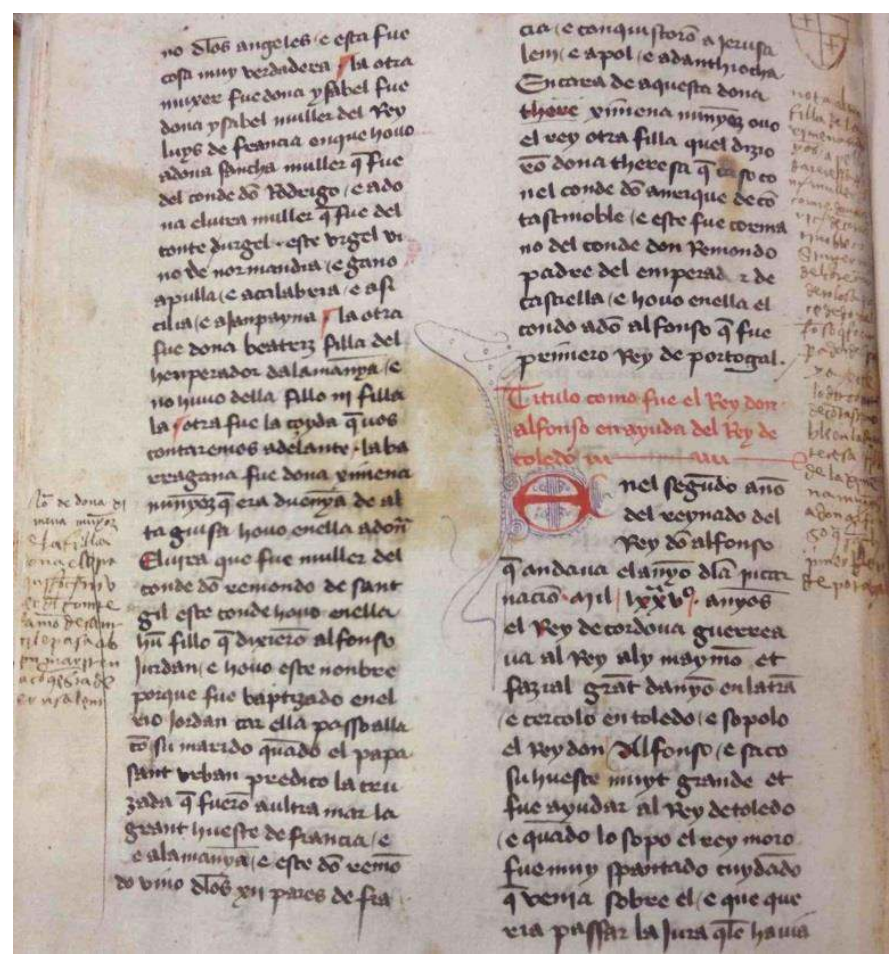

Fol. $48 \mathrm{v}^{\circ}$

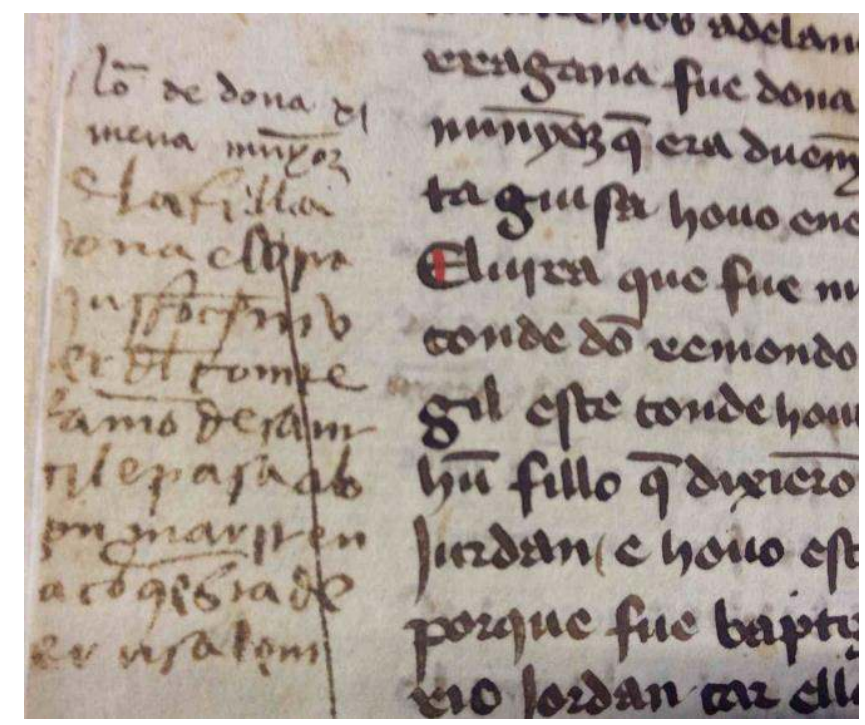

Détail du fol. $48 v^{\circ}$

On rencontre, enfin, un autre exemple de commentaire marginal accompagné de blasons au fol. $106 r^{\circ}$ où est rapporté le discours que prononce le roi Alphonse VI lors des cortès de Tolède pour défendre le Cid face aux infants de Carrión qui revendiquent la supériorité de leur naissance sur celle de Rodrigue. Dans ce passage, réapparaissent les noms d'«Eluira Munyoz» et de "Theresa Munyoz» signalés avec les mêmes blasons (à une variante près) et le commentaire s'ouvre sur ces mots : « mira como lo rey don Alfonso describia lo noble linatge del Sid Roi Dias Campeador desde los dos alcaldes Mateu Rasura et Lain Caluo... ». 


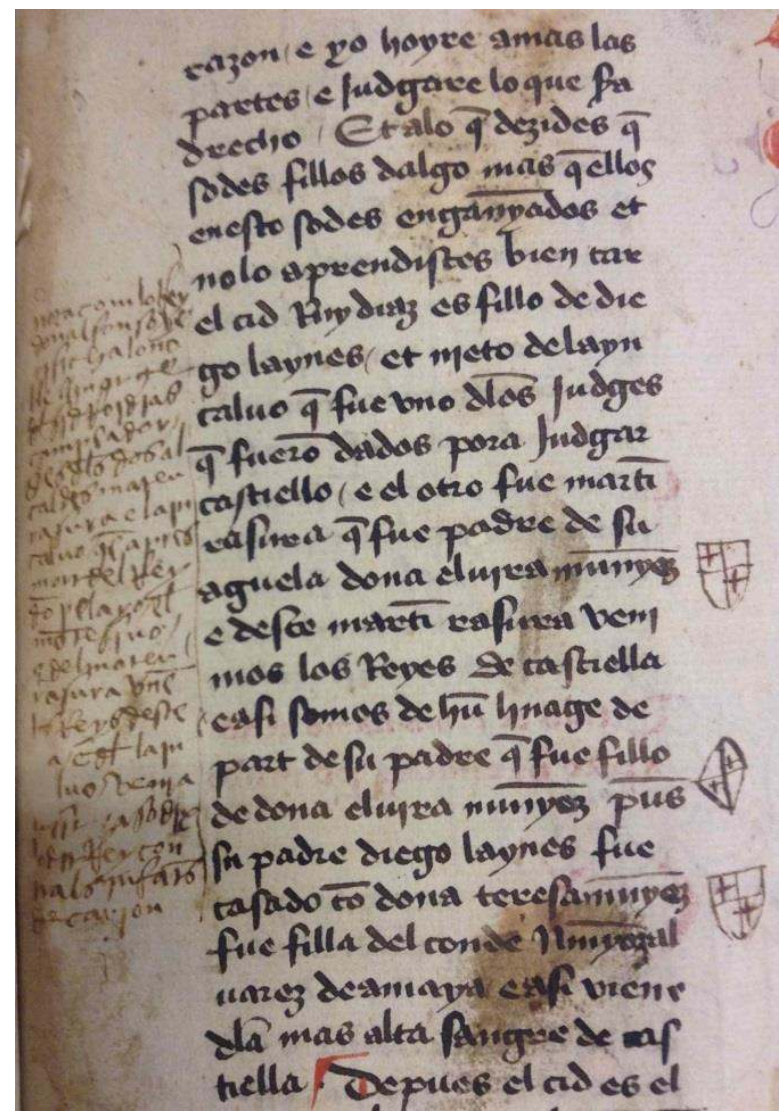

Fol.106r ${ }^{\circ}$

On remarquera que la dimension lignagère de ces annotations et de ces dessins est en parfait accord avec les propos de la chronique qui retrace, à travers le parcours du Cid, l'ascension des membres de l'aristocratie chevaleresque en valorisant le service royal. Les autres occurrences de ce type d'ajouts marginaux soulignent en effet la présence constante, dans l'entourage des monarques, d'hommes portant le nom de «Munyoz » ${ }^{26}$. C'est ce que l'on peut voir au fol. $61 r^{\circ}$ où sont énumérés, aux côtés du roi d'Aragon, les «altos hombres» que le Cid a affrontés en terres aragonaises :

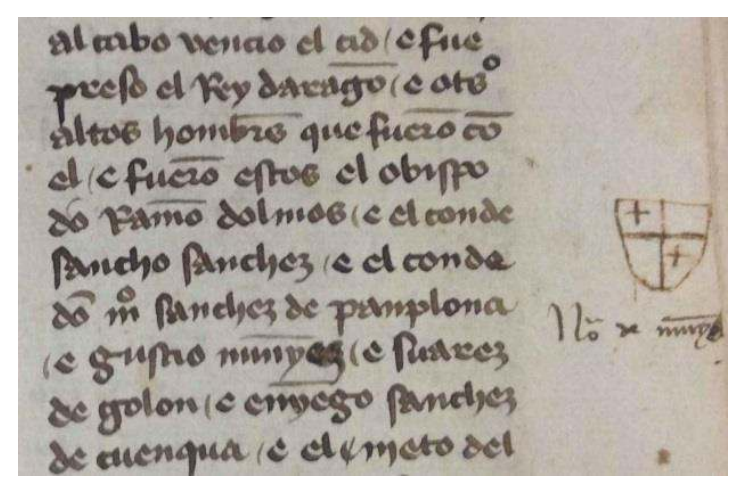

Fol. $61 \mathrm{r}^{\circ}$

${ }^{26}$ Outre les exemples qui sont cités dans le corps du texte, on pourra observer aussi ceux qui se trouvent aux folios $155 \mathrm{v}^{\circ}, 160 \mathrm{v}^{\circ}$ et $161 \mathrm{v}^{\circ}$ ainsi qu'au folio $147 \mathrm{v}^{\circ}$ où l'on trouve d'ailleurs un dessin représentant le chevalier, nommé «Pero Nunyez» dans le texte, emmenant sur son cheval le jeune Alphonse VIII de Castille pour le libérer de l'emprise de son oncle Ferdinand II de León. 
Les marginalia du manuscrit Esp 220 de la Bibliothèque Nationale de France présentent donc deux caractéristiques principales. Leur dimension religieuse renvoie, d'une part, à un milieu clérical et leur dimension lignagère révèle, d'autre part, des intérêts d'ordre aristocratique ${ }^{27}$. En outre, si la lecture qu'elles donnent du texte nous amène en terres aragonaises, certains éléments marginaux nous portent vers Valence et ses environs. On soulignera, enfin, que ces annotations ne sont pas toutes écrites de la même main. Elles se distinguent les unes des autres par une encre plus ou moins claire et par une écriture différente. En effet, certaines présentent une écriture proche de celle du manuscrit alors que d'autres sont plus tardives, ce que l'on remarque en observant par exemple la forme des « $\mathrm{d}$ », des « $\mathrm{r}$ » et des «s ». On comparera sur ce point les reproductions des folios $63 \mathrm{v}^{\circ}$ et $13 \mathrm{r}^{\circ}$ ou $48 \mathrm{v}^{\circ}$, mais on en voudra pour preuve cet autre exemple que l'on trouve au folio $22 \mathrm{r}^{\circ}$. Le récit de l'adoubement du Cid y est signalé par un trait vertical surmonté de trois points accompagné d'un commentaire stipulant « como el Cid Ruy Dias se fizo cauallero » qui pourrait être l'œuvre du copiste lui-même, comme le montrent la couleur de l'encre et l'écriture, mais on trouve aussi le mot nota abrégé, écrit avec une encre plus claire et des caractères plus gros.

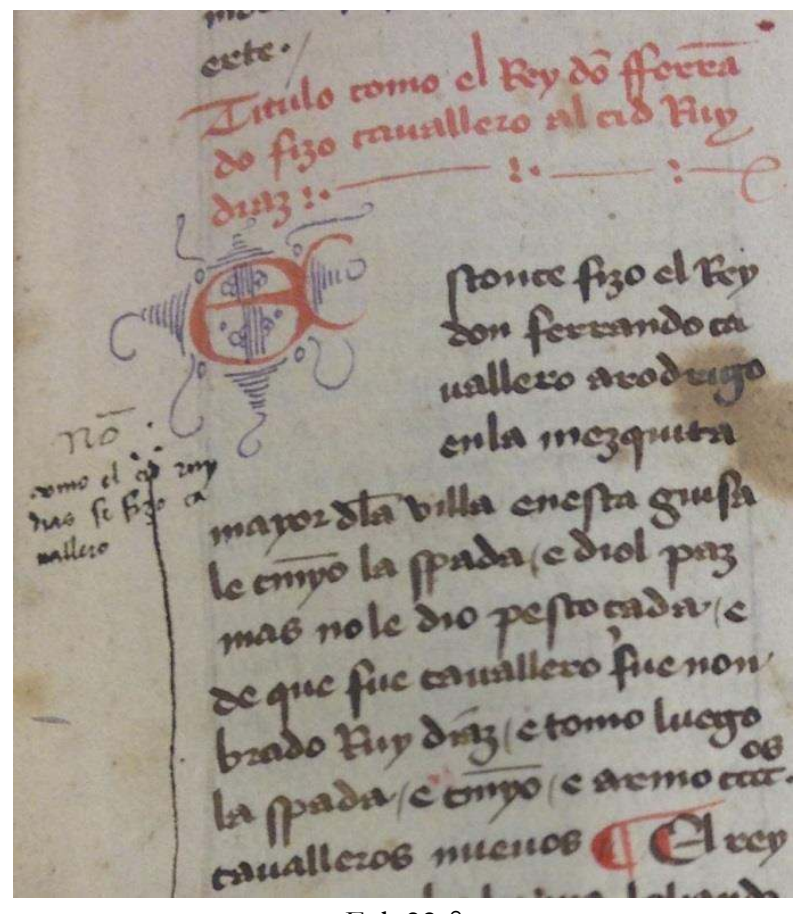

Fol. $22 \mathrm{r}^{\circ}$

Cependant, si l'on peut affirmer que les annotations marginales ont été réalisées par plusieurs mains, il n'en demeure pas moins qu'elles révèlent des préoccupations et des intérêts communs qui nous ramènent, comme nous allons le voir, aux membres d'un même lignage : les Sánchez Muñoz.

\footnotetext{
${ }^{27}$ Notons ici que l'on trouve même un cas où les deux aspects se conjuguent. En effet, au folio $24 \mathrm{v}^{\circ}$, en marge de l'épisode inspiré de la Chanson de Rodrigue et portant sur le tribut impérial que le pape et l'empereur tentent d'imposer au roi Ferdinand $\mathrm{I}^{\mathrm{er}}$, on trouve le commentaire suivant : « nota la fe qu'es entre los omenes del linatge ». Il s'applique à la lettre que le roi adresse, sur les conseils du Cid, à ses opposants, en leur demandant de renoncer au paiement de ce tribut et de les laisser mener leur lutte contre les Maures.
} 


\section{Les Sánchez Muñoz : promoteurs et lecteurs du Romanz del Cid}

C'est à Teruel, au XII siècle, que l'on trouve les premières traces des Sánchez Muñoz. En effet, la documentation nous indique qu'en 1171 le roi Alphonse II avait mené la conquête de la ville avec l'aide d'un groupe de chevaliers commandés par Sancho Sánchez Muñoz et Blasco Garcés de Marcilla ${ }^{28}$. Au cours des XIII ${ }^{\mathrm{e}}, \mathrm{XIV}^{\mathrm{e}}$ et $\mathrm{XV}^{\mathrm{e}}$ siècles, plusieurs membres de ce lignage issu de l'aristocratie chevaleresque occupèrent des postes de magistrats à Teruel, de sorte qu'ils figuraient parmi les familles les plus influentes de la ville. Le lignage comptait plusieurs branches, la plus importante étant celle des seigneurs et barons d'Escriche, qui nous intéresse tout particulièrement ici, et qui s'était distinguée par ses hauts faits aux côtés des monarques aragonais. Les chroniques rapportent en particulier que Jacques $\mathrm{I}^{\text {er }}$ aurait bénéficié de leur aide pour la conquête de Valence ${ }^{29}$. Or on trouve surtout, au sein de cette branche, un dénommé Gil Sánchez Muñoz y Carbón, né à Teruel en 1370 et surnommé le « Doncel », qui nous renvoie aux dernières années du Grand Schisme d'Occident.

En effet, Gil Sánchez Muñoz n’était autre que l’antipape Clément VIII qui avait succédé au pape de Luna Benoît XIII en 1423 et qui, comme son prédécesseur, vivait retiré au château de Peñiscola ${ }^{30}$. Dans les premières années du XV $\mathrm{XV}^{\mathrm{e}}$ siècle, Gil Sánchez Muñoz avait été chanoine de Valence puis vicaire général du diocèse de Valence. Si Alphonse V le Magnanime avait appuyé sa nomination en tant que pontife face à Martin V qui, lui, était soutenu par le roi de France, en avril 1429, date de la composition de notre manuscrit, il vivait les dernières heures de son pontificat. On sait en effet qu'en mai 1429, Alphonse V, qui avait fini par obtenir de Martin V son investiture en tant que roi de Naples, envoya plusieurs ambassadeurs à Peñiscola pour demander à Clément VIII de renoncer à sa dignité, ce qui fut fait le 26 juillet 1429. Parmi ceux qui, dans l'entourage de l'antipape, durent aussi abandonner leurs charges, figuraient trois cardinaux dont son neveu, Gil Sánchez Muñoz le Jeune, qui était d'ailleurs à la tête du chapitre de Valence ${ }^{31}$. Clément VIII quitta donc le château de Peñiscola, se dirigeant d'abord vers Valence, sans doute en compagnie, notamment, de Gil Sánchez Muñoz le Jeune ${ }^{32}$, et continua de jouir des rentes de ses charges ecclésiastiques avec l'accord d'Alphonse V et du pape Martin V. Ce dernier le nomma évêque de Majorque le 26 août 1429, mais ce n'est qu'en 1433 que Gil Sánchez Muñoz occupa le siège épiscopal. En effet, en octobre 1429, le cardinal Pierre de Foix, qui avait été nommé légat en Aragon pour mettre fin à la papauté de Peñiscola, avait placé à la tête de l'évêché de Majorque, avec le soutien du roi Alphonse V, un bénédictin du nom de Galcerán de Albert qui, malgré les pressions de Martin V, ne consentit à quitter les lieux qu'en avril 1431, au moment où Alphonse V se rangea du côté du pape ${ }^{33}$. Gil Sánchez Muñoz finit donc ses jours à Palma de Majorque, où il mourut le 28 décembre 1446.

\footnotetext{
${ }^{28}$ Là-dessus, vid. MUÑOZ GARRIDO, Vidal, « El linaje de los Sánchez Muñoz en Teruel (1170-1500) », p. 263-264.

${ }^{29}$ Cf. NAVARRO ESPINACH, Germán, « Muñoces, Marcillas y otras familias dominantes en la ciudad de Teruel (1435-1500)», p. 735.

30 Sur ce personnage, vid. GARCÍA MIRALLES, Fray Manuel, «La personalidad de Gil Sánchez Muñoz y la solución del Cisma de Occidente », Teruel, 12 (1954), p. 63-122 et NAVARRO ESPINACH, Germán et VILLANUEVA MORTE, Concepción, « Gil Sánchez Muñoz (1370-1447), el antipapa Clemente VIII : documentación inédita de los archivos de Teruel », Anales de la Universidad de Alicante. Historia Medieval, 15 (2006-2008), p. 239-254.

31 Vid. TOUZEAU, Gérard, Benoît XIII. Le trésor du pape catalan, Perpignan, Mare Nostrum, 2009, p. 202-203 et 205.

32 En 1430, Gil Sánchez Muñoz le Jeune était encore à la tête du chapitre de Valence (cf. ibid. p. 205). Il fut aussi archidiacre de Sagonte et protonotaire du pape et reçut divers bénéfices ecclésiastiques jusqu’à sa mort en 1471 ( $c f$. NAVARRO ESPINACH, G. et VILLANUEVA MORTE, C., «Gil Sánchez Muñoz (1370-1447), el antipapa Clemente VIII... », p. 250).

${ }^{33}$ Voir TOUZEAU, G., op. cit., p. 256-258.
} 
Plusieurs éléments de la biographie de Gil Sánchez Muñoz présentent donc des coïncidences avec les illustrations et les annotations marginales du manuscrit Esp 220 et laissent supposer qu'il pourrait être le promoteur mais aussi l'un des lecteurs et commentateurs de ce Romanz del Cid. Ainsi s'expliqueraient l'intérêt manifesté dans le manuscrit pour les épisodes miraculeux et pour le premier évêque de Valence, la précision apportée au château de Cebolla et la présence du mot «cisma» dans la marge. De même, le relevé systématique du patronyme Muñoz serait-il lié à l'histoire de cet homme et à celle des membres de son lignage. En effet, il convient d'ajouter qu'à cette période, les Sánchez Muñoz avaient tout intérêt à évoquer le prestige et les hauts faits de leur lignage pour faire valoir leurs droits auprès de la monarchie aragonaise puisqu'ils avaient perdu le titre de barons d'Escriche que détenait alors, depuis la fin du XIV siècle, une autre famille influente de Teruel, les Marcilla, leurs ennemis de toujours ${ }^{34}$.

Mais que dire de Gaspar Johan Sánchez Muñoz, qui fut, tel qu'on peut le lire à la fin du manuscrit, propriétaire de ce livre ? Unique représentant de la huitième génération de la branche des seigneurs d'Escriche, il était le fils de l'aîné des neveux de Clément VIII, Pedro Sánchez Muñoz y Marín de Urríes (1430-1483), et de Catalina Sánchez Gamir ${ }^{35}$. Il avait poursuivi la lutte entreprise par sa famille contre les Marcilla afin de récupérer Escriche, ce qui s'était soldé par un succès puisqu'il avait finalement obtenu, selon un document royal signé par Charles ${ }^{\text {er }}$ le 22 avril 1538, le titre de baron et seigneur d'Escriche. Mais surtout, Gaspar Johan Sánchez Muñoz avait hérité, par son père, de l'ensemble des biens de la famille et, notamment, de ceux de son grandoncle Clément VIII, tel que l'atteste un inventaire réalisé à Teruel et daté de $1484^{36}$. Ce document, conservé aujourd'hui à la Bibliothèque de Catalogne sous la côte 231, fut rédigé à la mort de Pedro Sánchez Muñoz survenue à Majorque le 24 novembre 1483. Il était destiné à répertorier de façon claire et complète tout ce qui revenait au jeune Gaspar Johan, alors seulement âgé d'un an. Or, il contient non seulement des annotations marginales qui montrent comment Gaspar Johan et ses descendants prirent soin de tout vérifier, mais il nous apprend aussi que Pedro Sánchez Muñoz était l'auteur d'un récit chevaleresque intitulé Letres de la batalla con mossén Pachs et qu'il laissait à son fils un coffre contenant plus de cent de livres ${ }^{37}$. Parmi ces livres figurait un «libro, cubiertas blanquas, en paper, e romanz, storiado, intitulado el Ciriodiaz. $\gg^{38}$ qui fut aussi répertorié dans l'inventaire de ses biens que réalisa à son tour Gaspar Johan en 1530 selon les termes suivants : «la corónica del Cit Ruy Diaz que ganó a Valencia, en romance » ${ }^{39}$.

Le manuscrit Esp 220 conservé à la Bibliothèque Nationale de France serait donc bien cette chronique rapportant les hauts faits du Cid que Pedro Sánchez Muñoz transmit à son fils Gaspar Johan. Du reste, comme l'indiquait ce dernier dans son inventaire, dans sa bibliothèque figuraient aussi «las Corónicas de Espanya que hizo el arcobispo don Rodrigo » ${ }^{40}$ qui pourraient correspondre à la référence aux «V quadernos de las Ystorias de Espanya » ${ }^{41}$ que l'on trouve dans l'inventaire de 1484. Ainsi l'annotation marginale renvoyant à l'œuvre de l'archevêque de Tolède Jiménez de Rada évoquée précédemment dans cette étude ferait-elle des Sánchez Muñoz les principaux lecteurs du manuscrit Esp 220.

\footnotetext{
34 Sur ces deux familles et leur rivalité, vid. NAVARRO ESPINACH, G., « Muñoces, Marcillas... », p. 734-750.

35 Sur Gaspar Johan Sanchez Muñoz, voir NAVARRO ESPINACH, G., « Muñoces, Marcillas... », p. 741-743.

${ }^{6}$ Cf. WITTLIN, Curt J., "Un inventario turolense de 1484 : Los Sánchez Muñoz, herederos del papa Clemente VIII », Revista Archivo de Filiogía Aragonesa, 18-19 (1976), p. 187-215.

37 Ibid., p. 188, 189 et 191.

38 WITTLIN, Curt J., «Les manuscrits dits 'Del Papa Luna' dans deux inventaires de la bibliothèque de Gaspar Johan Sánchez Munyoz à Teruel », Estudis romànics, 11, 1962, p. 11-32, p. 18. Sur ces deux inventaires, on pourra consulter aussi MONFRIN, Jacques, «La bibliothèque Sánchez Muñoz et les inventaires de la bibliothèque pontificale à Peñíscola ", in Studi di bibliografia e di storia in onore di Tammaro di Marinis, Verona, Biblioteca Apostolica Vaticana, 1964, 4 vol., vol. 3, p. 229-269.

39 WITTLIN, Curt J., « Les manuscrits dits 'Del Papa Luna'... », p. 27.

${ }^{40}$ Loc. cit.. Gaspar Johan mentionne aussi « las Corónicas de Espanya y de los godos, en romance ».

${ }^{41}$ Ibid., p. 25.
} 
Tout comme son père, Gaspar Johan Sánchez Muñoz était d'ailleurs passionné par les livres. Il avait ainsi enrichi la bibliothèque héritée de son père de ses livres d'école, d'une douzaine de textes humanistiques et de quelques livres imprimés ${ }^{42}$. En outre, il rédigea un journal relatant, à partir de 1500, les événements marquants survenus à Teruel ${ }^{43}$. Et surtout, détail important, il avait coutume d'annoter les livres qu'il lisait! Or en comparant certaines des annotations marginales du manuscrit avec l'inventaire de 1530 conservé à la Bibliothèque de Catalogne sous la côte 234, il semblerait que l'on puisse reconnaitre l'écriture de Gaspar Johan Sánchez Muñoz ${ }^{44}$.

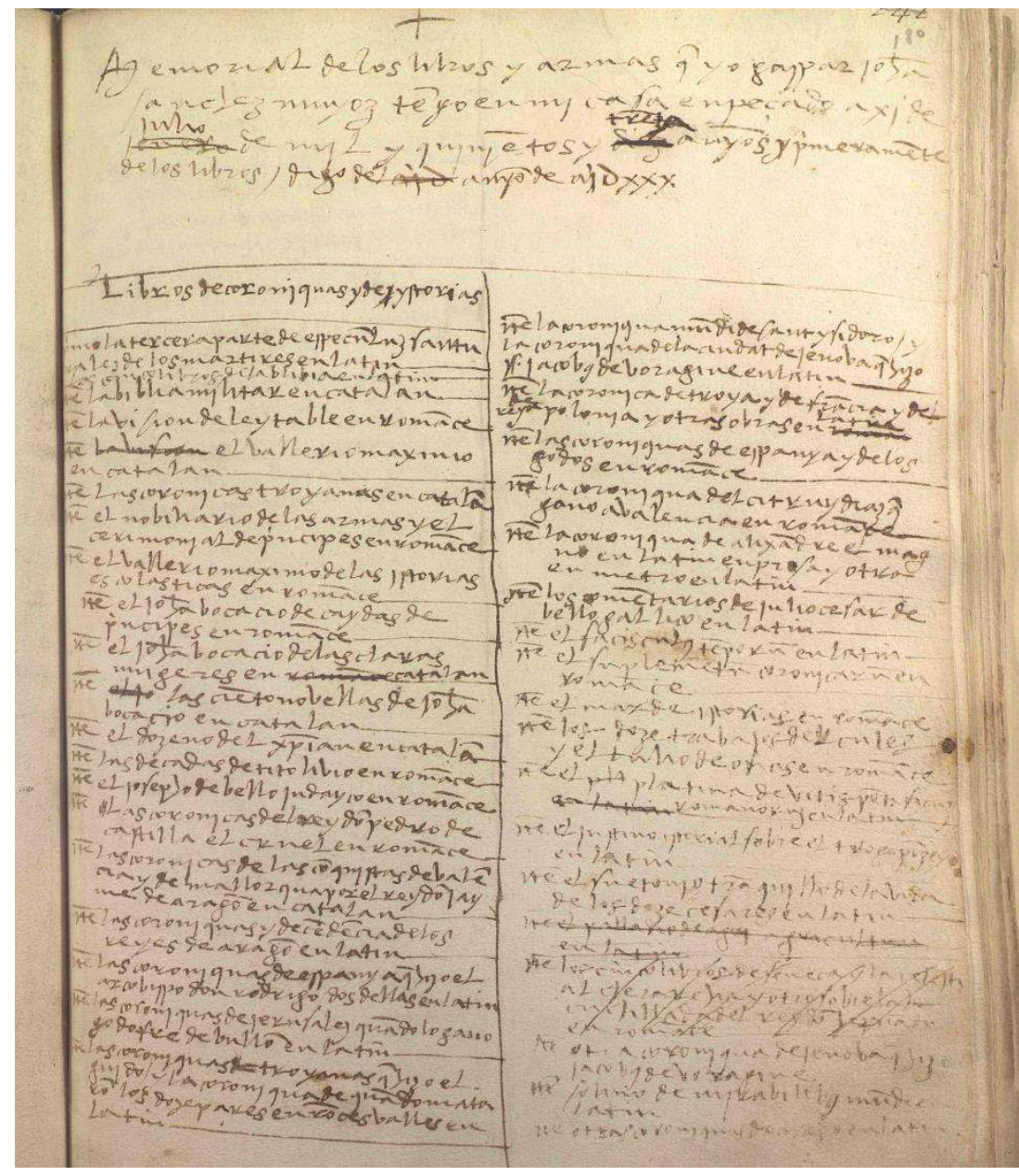

Extrait de l’inventaire de 1530 rédigé par Gaspar Johan Sanchez Muñoz (ms. 234, Biblioteca de Catalunya)

L'étude des interactions entre le texte, les images et les annotations marginales du manuscrit Esp 220 de la Bibliothèque Nationale de France nous offre donc des informations de premier ordre sur le cheminement du $«$ livre au lire ${ }^{45}$ et sur la part active de la lecture au sein de

42 Ibid., p. 12.

${ }^{43}$ Loc. cit.. Pour une consultation du journal de Gaspar Johan, voir LLABRÉS Y QUINTANA, Gabriel, «Diario turolense de la primera mitad del siglo XVI, por D. Juan Gaspar Sánchez Muñoz ", Boletín de la Real Academia de la Historia, 27 (juillet-septembre 1895), p. 5-75.

${ }^{44}$ Je remercie ici la responsable de la section des manuscrits de la Biblioteca de Catalunya, Mme Anna Gudallol Torelló, de m’avoir fait parvenir cette reproduction.

45 Cette expression est tirée du titre d'un article de Roger Chartier qui, s'il traite presque essentiellement du livre imprimé, n'en comporte pas moins des réflexions intéressantes sur les pratiques et les propos de la « mise en texte » et de l'acte de lecture, qui s'appliquent fort bien aux manuscrits médiévaux [ $c f$. CHARTIER, Roger, «Du livre au 
l'aristocratie chevaleresque aragonaise des $\mathrm{XV}^{\mathrm{e}}$ et $\mathrm{XVI}^{\mathrm{e}}$ siècles. Elle nous permet de retracer l'histoire du manuscrit du Romanz del Cid, de sa composition à sa réception, et d'imaginer qu'il fut composé à la demande de Clément VIII, soit au château de Peñiscola, ce qui signifierait qu'il échappa aux biens que l'antipape fut tenu d'abandonner au moment de sa renonciation ${ }^{46}$, soit au sein de la cathédrale de Valence ${ }^{47}$, et qu'il fut ensuite transmis à Pedro Sánchez Muñoz qui le légua à son fils Gaspar Johan. L'intérêt porté aux récits de miracles et aux hommes d'église, et en particulier à l'évêque Jérôme de Valence, renverrait donc au commanditaire du manuscrit et à ses premiers lecteurs. Quant aux annotations mettant en lumière les exploits de l'aristocratie chevaleresque et relevant systématiquement le patronyme Muñoz, elles seraient l'œuvre, sinon de Clément VIII ou de Gil Sánchez Muñoz le Jeune, du moins de son autre neveu Pedro et de son petit-neveu Gaspar Johan, qui étaient tous deux de grands lecteurs et qui avaient fort intérêt, face aux Marcilla, à vanter les mérites et les prouesses de leur lignage afin de récupérer la seigneurie d'Escriche.

Il resterait, néanmoins, un dernier point à éclaircir : comment le manuscrit Esp 220 se retrouva-t-il dans les fonds de la Bibliothèque Nationale de France ? Mais c'est là une autre histoire qui se situe en marge du propos de cette étude.

lire ", in Id., Pratiques de la lecture, (1 1re éd., Paris, Rivages, 1985), 3e éd., Paris, Petite Bibliothèque Payot, 2003, p. 81-117 et notamment p. 104-109].

${ }^{46}$ En 1429, le cardinal Pierre de Foix, légat du Saint-Siège, prit possession du château au nom du pape Martin V et dressa un inventaire de tous les biens qui s'y trouvaient (cf. MONFRIN, Jacques, art. cit., p. 230). Cet inventaire a été édité par MARTÍ DE BARCELONA, P., «La Biblioteca papal de Penyiscola. Inventari dreçat pel cardenal Pere de Foix », O. M., 1429, in Estudis franciscans, 28 (1922), p. 336-341, 420-436 et 30 (1923), p. 88-94 et 266-272.

${ }^{47}$ La présence et l'influence des Sánchez Muñoz au sein de la cathédrale de Valence remontent d'ailleurs à la fin du XIVe siècle, comme l'atteste la fondation de la chapelle des "Muñoces », dédiée à sainte Anne, qui fut réalisée à la demande d'un oncle de Clément VIII, lui-même chanoine de Valence et appelé lui aussi Gil Sánchez Muñoz ( $c f$. NAVARRO ESPINACH, G. et VILLANUEVA MORTE, C., «Gil Sánchez Muñoz (1370-1447), el antipapa Clemente VIII... », p. 247). 